\title{
Rechtfertigungsordnungen des Alter(n)s
}

\author{
Von Stefanie Graefe und Stephan Lessenich
}

Zusammenfassung: Innerhalb der normativen Ordnung des Wohlfahrtstaates hat sich ein bemerkenswerter Wandel vollzogen: Galt das Alter lange Zeit als eine - wohlfahrtsstaatlich zu versorgende - Art „Rest“-Lebensphase, die auf die Normalerwerbsbiographie folgt, so erscheint es nunmehr einerseits als produktive Ressource zur Bewältigung des demographischen Wandels, andererseits als biographisches Potenzial, das von den alternden Individuen auch in ihrem eigenen Interesse produktiv genutzt werden kann und sollte. Normen, die bislang vor allem für die mittlere Lebensphase galten, werden so auf spätere Lebensphasen ausgedehnt. Der diskursiven Aufwertung zum Trotz existieren zwischen älteren Menschen vielfältige symbolische und materielle Ungleichheiten - und damit auch Formen sozialer Distinktion. Vor dem Hintergrund der Wende im normativen Diskurs ums Alter(n) sind Distinktionsgewinne vor allem für all jene Älteren zu erwarten, deren Lebensführung bereits den aktivitäts- und produktivitätsorientierten Verhaltensidealen entspricht. Auf der Grundlage einer qualitativ-empirischen Untersuchung geht der Beitrag der Frage nach, ob und wie sich in subjektiven Alter(n)sdeutungen eine sozialstrukturell in typischer Weise differierende Nähe zum Leitbild des produktiven Alter(n)s ausmachen lässt. Im Ergebnis zeigt sich, dass sich vorrangig in bildungsbürgerlichen Milieus eine deutliche - praktische und symbolische - Nähe zu produktivistischen Lebensführungsmustern und Alter(n)sbildern findet. Die diskursive Aufwertung „des“ Alters lässt sich davon ausgehend auch als indirekte Stabilisierung der kulturellen Hegemonie gehobener Mittelklassenmilieus interpretieren.

\section{Einleitung}

Die Frage, was ein ,gutes“ Alter ausmacht, wie man in ,angemessener“ Weise altert und wie alte Menschen ,altersadäquat“ leben, lässt sich nicht unabhängig von jener moralischen Ökonomie beantworten, die - in historisch je spezifischer und nationalkulturell geprägter Form durch den modernen Wohlfahrtsstaat etabliert wird. Der Befund, dass Alter(n) längst kein „sozialstrukturell [...] eindeutig konstituierter und von den anderen Lebensphasen abgegrenzter Bereich mehr" (Backes et al. 2001: 7) ist, gilt gegenwärtig mehr denn je. Dabei markiert in jüngerer Zeit vor allem die Entdeckung des ,,jungen“, ,erfolgreichen“, ,,aktiven“ und ,produktiven" Alter(n)s ${ }^{1}$ einen historischen Wendepunkt im wohlfahrtsstaatlichen Altersnormativ. Die Lebensphase Alter ist - so jedenfalls das gegenwärtige sozialpolitische und -gerontologische Versprechen - nicht mehr bloß zu verwaltendes Residuum einer produktiven Normalerwerbsbiografie, sondern wird als gesellschaftlich notwendige Ressource zur Bewältigung der Krise des Wohlfahrtsstaates und des demografischen Wandels sowie als eine Lebensphase mit neuen individualbiografischen Gestaltungsmöglichkeiten thematisiert.

Die allgemeine Anrufung der (gegenwärtig wie zukünftig) „Alten“ als gesellschaftliche Ressource an sich und für sich (Denninger et al. 2012) sollte jedoch nicht darüber hinwegtäuschen, dass soziale - symbolische, strukturelle und materielle - Ungleichheiten vor dem Alter nicht Halt machen. Dabei stellt das Alter(n) für Konzepte sozialer Ungleichheit eine besondere Herausforderung dar - und die Frage, ob von einer lebenslangen Kontinuität sozialer Ungleichheit oder von der Verschärfung von Ungleichheiten im Alter auszugehen ist, ist soziologisch umstritten (Kohli et al. 2005: 318ff). Unstrittig hingegen ist, dass die Verteilung von Mortalität und Morbidität - und damit die schlichte Dauer des individuell zu erlebenden Alters

1 Zur Genese und Differenz der genannten Konzepte vgl. ausführlich van Dyk / Lessenich 2009. Für das Thema des vorliegenden Beitrags ist weniger ihre Differenz als vielmehr ihr gemeinsamer Nenner entscheidend: die gesellschaftliche „Entdeckung“ der ,produktiven“ Ressourcen des Alter(n)s. 
- hochgradig mit vertikalen sozialen Ungleichheitsfaktoren wie Einkommen, Beruf und Bildungsstand korreliert (vgl. z.B. Richter / Hurrelmann 2007; Lampert et al. 2005). Je älter eine Person ist, umso relevanter ist ihre sozialökonomische Ressourcenausstattung - nicht nur im Hinblick auf Gesundheit und Lebenserwartung, sondern auch in Bezug auf Wohnsituation und soziale Netzwerke (Kohli et al. 2005: 332), subjektives Wohlbefinden und Selbstbewusstsein (McMullin / Cairney 2004). Diese Differenzen bleiben im sozialpolitischen Diskurs um die Neuentdeckung der Produktivität des (jungen) Alters jedoch meist unterbelichtet: Der/die sportliche, konsumfreudige, lebenslang lernende und sozial engagierte junge Alte scheint sozialstrukturell neutral zu sein.

Die Frage, ob sich die neue Sichtbarkeit der so genannten ,jungen Alten“ der gesellschaftlichen Hegemonie spezifischer sozialer Klassenmilieus (Vester et al. 2001) verdankt, wird bislang vor allem in der angelsächsischen Sozialgerontologie debattiert. Prominent vertreten etwa Gilleard und Higgs: „Class [...] is neither an explanation for, nor the structural equivalent of the third age." (Gilleard / Higgs 2002: 374) Damit grenzen sie sich gegen Ansätze ab, die die neue gesellschaftliche Wertschätzung des sogenannten „Dritten Alters“, also der Altersgruppe „50 plus“ samt ihrer produktiven und konsumtiven Potenziale, vorrangig auf die im Vergleich zu früheren Generationen objektive materielle Besserstellung dieser Gruppe - und damit auf ökonomische Produktions- und Verteilungsverhältnisse - zurückführen. Gilleard und Higgs betonen demgegenüber, die gesellschaftliche „Entdeckung“ des Dritten Lebensalters verdanke sich weniger ökonomischen Faktoren als vielmehr generationsspezifischen Lebens- und Konsumstilen. Die heutigen Älteren repräsentierten ein ,generational field who's origins lie in the ,youth culture' of the 1950s and 1960s" (Gilleard / Higgs 2005: 151).

Aufgrund der unterschiedlichen demografischen Entwicklungen nach dem Zweiten Weltkrieg sind die Kohorten der US-amerikanischen ,third agers“ deutlich älter als die hierzulande sogenannten ,jungen Alten“. Doch auch für Deutschland lässt sich fragen, ob die Diskursfigur des ,jungen Alters“ nicht vorrangig die Werte einer „Post-68er“-Kultur - Engagement, Autonomie und Individualität - repräsentiert. Allerdings muss die kulturelle Sichtbarkeit der gegenwärtigen ,jungen Alten“ nicht entweder mit ihrem generations- bzw. kohortenspezifischen Lebensstil oder ihrer Zugehörigkeit zu spezifischen Klassenmilieus erklärt werden. Vielmehr ist anzunehmen, dass generationstypische Lebensstile nur dann diskursiv und sozialpolitisch relevant werden, wenn sie von Angehörigen hegemonialer gesellschaftlicher Gruppen repräsentiert werden. Versteht man „class“ als „dynamic process which is the site of political struggle, rather than a set of static and empty positions waiting to be filled by indicators such as employment and housing“ (Lawler 2005: 430), so bedeutet dies, dass hegemoniale Werte und Lebensstile nicht nur zwischen, sondern auch innerhalb von Generationen entlang verschiedener sozialer Klassenmilieus umkämpft sind. Alter kann (und muss) in beiderlei Hinsicht als ,,soziale Dimension der Gesellschaftsstruktur und zugleich als normative und symbolische Dimension“ (Amann / Kolland 2008: 32; vgl. von Kondratowitz 2009) begriffen werden womit sich die Frage stellt, wie beide Aspekte miteinander vermittelt sind (vgl. Amrhein 2008).

Dieser Frage wollen wir im Folgenden nachgehen. Uns interessiert der Zusammenhang von subjektiven Deutungen des Alter(n)s und symbolisch-normativen Dimensionen der Lebensführung sowie (damit einhergehend) die Nähe bzw. Distanz subjektiver Alter(n)sdeutungen zum diskursiv hegemonialen Deutungsangebot des ,produktiven Alter(n)s“. ${ }^{2}$ Inwiefern ist die subjektive Alter(n)serfahrung durch soziale Normen (vor)strukturiert (Kolland 2010: 77), die dem (altersunabhängigen) Lebensstil spezifischer Sozialmilieus korrespondieren? Dabei greifen wir auf die Ergebnisse einer qualitativen Studie zu subjektiven Alter(n)sbildern und Alter(n)serfahrungen von Menschen in der zweiten Lebenshälfte in Westdeutschland zurück

2 Hier im Sinne einer begrifflichen Klammer für „erfolgreiches“, ,aktives“ und „produktives“ Alter(n) zu verstehen, vgl. Fn. 1. 
(3) ${ }^{3}$ Vorab rekapitulieren wir die Geschichte der symbolischen und materiellen Reproduktion sozialer Hegemonie, sprich: des Normativs der „Bürgerlichkeit“, im spätmodernen ,aktivierenden" Wohlfahrtsstaat, der mittlerweile auch die Formen der Vergesellschaftung des Alters prägt (2). Abschließend fassen wir zentrale Ergebnisse der konzeptionellen und empirischen Befunde zusammen (4).

\section{Produktives Alter(n) und neue Bürgerlichkeit im „aktivierenden Sozial- staat $^{\text {"6 }}$}

Die gesellschaftlich herrschenden Rechtfertigungsordnungen des Alter(n)s sind in der fortgeschrittenen Moderne eng mit der normativen Ordnung des Wohlfahrtsstaats verbunden. Als komplexes System der politischen Regulierung und Verteilung sozialer Güter, Positionen und Lebenschancen ruht der moderne Wohlfahrtsstaat, neben seiner funktionsnotwendigen ökonomischen Basis, auf einem kulturellen Fundament sozialmoralischer Normen, Ressourcen und Intuitionen. Wer im wohlfahrtsstaatlichen Arrangement was und wie viel von wem bekommt, sei es nun im Sinne faktisch-messbarer oder aber bloß vermuteter und unterstellter Umverteilungsbeziehungen, wird im Prozess gesellschaftlicher Aushandlung und politischer Ausgestaltung der sozialen Ungleichheitsordnung immer mit der entscheidenden Warum-Frage verknüpft: Stets geht es darum, welche wohlfahrtsstaatlich organisierten Ressourcenflüsse zwischen Individuen, Haushalten und Kollektiven aus welchen Gründen akzeptabel sind und als legitim anerkannt werden - und welche wohlfahrtsstaatlich konstituierten Sozialkategorien daher für ihre relative Positionierung im Verteilungsraum gesellschaftliche Geltung reklamieren können. Dieser sozial-politische Geltungskonflikt wird auf den unterschiedlichsten Feldern wohlfahrtsstaatlicher Vergesellschaftung ausgetragen, womit sich eine strukturelle Dynamik ineinander verschränkter, interagierender Anerkennungs- und Verteilungskämpfe zwischen Klassen und Geschlechtern, ethnischen Gruppen und Generationen einstellt.

In einer sicherlich nicht unumstrittenen, aber historisch wohl doch zulässigen Zuspitzung kann der klassenpolitische Konflikt als die strukturprägende Dimension wohlfahrtsstaatlicher Entwicklung gelten. Zumindest auf den ersten Blick paradoxerweise entsteht aus dem maßgeblich von den ,arbeitenden Klassen“ und deren Interessenorganisationen geführten und gegen die materiellen Privilegien des Besitzbürgertums gerichteten Kampf um soziale Berechtigung im Verlauf des 20. Jahrhunderts, und insbesondere im Zeichen der langen Prosperitätsphase nach dem Zweiten Weltkrieg, ein zunehmend ausgebauter Sozialstaat, dessen Institutionen und Interventionen allerdings durchaus „bürgerliche“ Interessen bedienen. Letztlich in allen Varianten des westlichen Wohlfahrtskapitalismus, insbesondere aber im deutschen Modell einer sozialpolitisch regulierten Marktwirtschaft, entpuppt sich die Etablierung und Expansion des Wohlfahrtsstaats als ein Mittelschichtsprojekt. Es ist das System der Garantie sozialstaatlicher Transfereinkommen auch in den Wechselfällen der Lohnarbeiterexistenz und über diese hinweg (Achinger 1958), das die materiellen Errungenschaften des „Wirtschaftswunders" für zunehmend breitere Bevölkerungsteile auf Dauer gestellt und deren sozialen Aufstieg gleichsam verrechtlicht hat. Und es sind (bzw. waren) die Programme und Programmierungen des Sozialstaats - von der Eigenheimzulage bis zur Pendlerpauschale, von der Ausbildungsförderung bis zur Pflegeversicherung, von der beitragsfreien Mitversicherung Familienangehöriger bis zum steuerrechtlichen Ehegattenprivileg, vom statussichernden Ar-

3 Die Studie wurde im Rahmen des von der VolkswagenStiftung geförderten interdisziplinären Forschungsprojektes „Zonen des Übergangs“ an der Universität Jena durchgeführt (http://www.soziologie.uni-jena.de/alternsforschung_zonendesuebergangs.html). Im Rahmen der soziologisch-qualitativen Teilstudie wurden rund 40 Personen westdeutscher Herkunft zwischen 45 und 95 Jahren nach subjektiven Vorstellungen und Erfahrungen des Alter(n)s befragt. Zum methodologischen und methodischen Vorgehen vgl. Graefe et al. 2011. Wir danken Silke van Dyk sowie zwei anonymen Gutachter/ innen für hilfreiche Hinweise beim Verfassen dieses Beitrags. 
beitslosengeld bis zur dynamischen Altersrente -, die den sozialhistorischen Siegeszug der gesellschaftlichen „Mitte“ und die gesellschaftsweite Verbreitung „,bürgerlicher“ Formen alltäglicher Lebensführung und biografischer Lebensplanung überhaupt möglich gemacht haben (Lessenich 2009 a: 20ff; Vogel 2009: 116ff). Dabei traten mit der Zeit insbesondere die akademisch geprägten, bildungsbürgerlichen Milieus als gesamtgesellschaftliche Meinungsbildner und soziale Taktgeber der Etablierung und des Wandels hegemonialer Lebensstilnormen auf. Die bildungsnahen Klassen der oberen Mittelschicht repräsentierten jene Vorstellungen eines ,gelungenen“ Lebens, an denen sich die breite Masse der ,arbeitnehmerischen Mitte“ in ihrem wohlfahrtsstaatlich beförderten sozialen Aufstiegsstreben orientierte (Vester 2005: 56ff).

Wenn man so will, beruht die historische Koalition von Mittelschichten und Wohlfahrtsstaat auf Wechselseitigkeit: Der ,sorgende Staat“ (de Swaan 1993) der Nachkriegszeit hat die Mittelschichten - sozialstrukturell wie soziokulturell - erst groß gemacht, und nur als ein Angebot für die „breite Mitte“ konnte ein großer, teurer Sozialstaat gesellschaftspolitisch auch mehrheitsfähig werden: „The consolidation of welfare states after World War II came to depend fundamentally on the political alliances of the new middle classes" (Esping-Andersen 1990: 31; Goodin / LeGrand 1987). Der Mittelschichtssozialstaat gibt einer meritokratischen Gerechtigkeitsvorstellung Ausdruck und Auftrieb, wonach sich Leistung - verstanden als marktförmige Verausgabung von Arbeitskraft - lohnen und nicht nur auf dem Markt selbst, sondern auch über lohnarbeitsvermittelte soziale Sicherungsansprüche angemessen honoriert werden soll. Im Sinne dieser spezifischen Moralökonomie (Thompson 1980) fördern sozialstaatliche Eingriffe und Einrichtungen direkt oder indirekt, explizit und implizit mittelschichtstypische Bildungsbiografien und Erwerbskarrieren, Haushalts- und Familienformen, Konsummuster und Siedlungsstrukturen. Der „,neue Mittelstand“ der höheren Angestellten und öffentlich Bediensteten, das Sozialmilieu der - zu großen Teilen im Auftrag des Wohlfahrtsstaats arbeitenden - oberen „Dienstklassen“ wird in diesem Kontext zum gesellschaftlichen Rollenmodell des modernen Wohlfahrtskapitalismus, die akademisch-verbeamtete service class zur normierenden Instanz und prototypischen Sozialfigur des middle class way of life der langen Nachkriegszeit (vgl. Schommer 2008: 35ff).

Dass sich dieses spezifische Arrangement formierender und - direkt oder indirekt - normierender Macht wohlfahrtsstaatlicher Institutionen herstellt, ist soziologisch keineswegs trivial und war historisch alles andere als vorentschieden. ${ }^{4}$ Die soziale Realität der Mittelstandsgesellschaft war und ist bis heute eine Frage und das Ergebnis gesellschaftlicher Auseinandersetzungen um die Gestalt und Gestaltung des Wohlfahrtsstaates - eine gesellschaftspolitische Konfliktkonstellation, in welcher dieser keineswegs neutrales Kampffeld konkurrierender Ideen und Interessen, sondern selbst Partei ist, indem er einen sozialen Raum relativer Verteilungspositionen konstituiert und darin vorzugsweise bestimmte Soziallagen mit materiellen Ressourcen und symbolischen Machtchancen ausstattet (Bourdieu 1998; vgl. Kuchler 2006: $7 \mathrm{ff}$ ). Im modernen Wohlfahrtsstaat, der mit der Vergabe sozialer Bürgerrechte operiert und sich strukturlogisch wie entwicklungsgeschichtlich durch die Ausweitung der politischen Garantie entsprechender Rechtspositionen auf tendenziell alle Staatsbürger/innen auszeichnet (Marshall 1963), dreht sich der sozialpolitische Kampf immer auch darum, ob und wie dieser (je nationale) Universalismus der Berechtigung gleichwohl einer sozialen Kontrolle unterworfen werden kann. Aus Sicht nicht nur der sozialpolitischen Steuerungsinstanzen, sondern auch der durch sie institutionell privilegierten bürgerlichen Milieus lautet die moralökonomische Gretchenfrage der wohlfahrtsstaatlichen Gesellschaftsformation demgemäß: Wie lässt

4 „The middle class, like any other social group, does not exist ready-made in reality. It must be constituted through material and symbolic struggles waged simultaneously over class and between classes; it is a historically variable and reversible effect of these struggles." (Wacquant 1991: 57). 
sich verhindern, dass die Etablierung und sachliche wie personelle Ausweitung sozialer Anspruchsrechte die Selbstbeschreibung der liberalen Bürgergesellschaft als einer Gemeinschaft arbeitsamer, leistungsorientierter, selbstverantwortlicher Individuen unterläuft und damit deren stabile Reproduktion langfristig untergräbt?

Die Sorge um die bürgerliche Moral im demokratischen Wohlfahrtsstaat, die Angst vor einem der individuellen „Eigenverantwortung“ enthebenden „Versorgungsstaat“, der Verdacht einer öffentlichen Förderung des Verfalls von Prinzipien und Praktiken ,gutbürgerlicher" Lebensführung sind ständige Begleiter der wohlfahrtsstaatlichen Entwicklung. Sie sind letztlich immer auch Medien der Sicherung privilegierter Lebenschancen und der Reproduktion symbolischer Machtpositionen, konkret: der sozialen Selbstbehauptung der oberen gegen die „nach oben“ drängenden unteren Mittelschichten, deren Aufstiegswille angetrieben werden soll, ohne die gesellschaftliche Statushierarchie zu gefährden. Insofern sind eine „möglichst systematische Verhaltenskontrolle und Verhaltenssteuerung [...] die zentralen sozialpolitischen Themen einer Gesellschaft, die sich als Bürgergesellschaft versteht" (Rieger 2003: 217; Hervorhebung im Original). Der strukturelle Kampf um die „Bürgerlichkeit“ der wohlfahrtsstaatlich Berechtigten lässt sich damit auch als „Klassenkampf“ der Lebensführungsmuster verstehen - ein kulturell-strukturelles Kampffeld, auf dem die bürgerlichen Kontrollimpulse allerdings keineswegs zu jeder Zeit auf gleiche Weise und mit gleicher Intensität zum Tragen kommen, und von dem das hier interessierende Alter als soziale Kategorie und individualbiografische Lebensphase bemerkenswerterweise für geraume Zeit ausgenommen war.

Zumindest über einige Jahrzehnte der Nachkriegsgeschichte hinweg waren die Sozialfigur des „Altersrentners“ und die Sozialkonstruktion eines „wohlverdienten Ruhestands“ zu - so zumindest der Anschein - festen Bestandteilen der wohlfahrtsstaatlichen Moralökonomie geworden: Den gleichsam ein Leben lang (berufs)tätigen Mitbürger/innen sollte, qua „Generationenvertrag“, auch im höheren Alter ein materiell gesicherter und damit nicht nur von Existenzsorgen, sondern auch von Erwerbs- und sonstigen Tätigkeitspflichten befreiter „Lebensabend" gewährleistet werden (Göckenjan 1993). Anders als die Erwerbs- oder auch die Ausbildungsphase wurde das „dritte“ Lebensalter - einmal erreicht - von sozialpolitischen Verhaltensnormierungen und gesellschaftlichen Erwartungshaltungen weitestgehend entkoppelt. Die im Zeichen ihrer ruheständischen Lebensführung als „Senioren“ adressierten Alten schienen, von den Mühen des Arbeitslebens entlastet und aller sonstigen öffentlichen Ansprüche enthoben, in der splendid isolation wohlfahrtsstaatlicher Versorgungslagen gut aufgehoben und damit nicht nur aus der Erwerbsarbeit, sondern auch aus den klassenpolitischen Kämpfen der Erwerbsgesellschaft entlassen. Das Alter konstituierte sich als eine Parallelgesellschaft scheinbar klassenloser Individuen - allerdings nicht auf Dauer.

Mit dem ,aktivierenden“ Umbau des Sozialstaats (Lessenich 2008) und dem damit intendierten, zumindest tendenziell auch schon vollzogenen Paradigmenwechsel von der „,konsumtiven“ zu einer „produktiven“ Sozialpolitik (Münch 2009) ist der Kampf um die Ausgestaltung des Bürgerstatus und um den Bürgerlichkeitsstatus der Sozialstaatssubjekte zuletzt neu entflammt - und zugleich die sozialpolitische Kampfzone ausgeweitet worden. In die strategische Umstellung der Sozialpolitik ,,von unproduktiven Formen der Abwicklung der Vergangenheit“ auf „,produktive Formen der Gestaltung von Zukunft“ (Münch 2009: 163) - von der „Versorgungs“- auf die „Investitions“-Logik - wird auch die Generation der Ruheständler/innen einbezogen. Dies zum einen, weil mit den ,jungen Alten“ eine, je nach sozialpolitischer Beobachterperspektive, Altersgruppe bzw. -kohorte identifiziert worden ist, die hinsichtlich ihres Einkommens, ihres Bildungsstands und ihrer gesundheitlichen Lage als durchaus (und durchweg) ressourcenstark beschrieben wird - Ressourcen, die es einer öffentlich zunehmend prominent werdenden Überzeugung zufolge zu nutzen gilt, wenn im Zeichen des demografischen Wandels aus dem ehedem kurzen Lebensabend des Sozialrentners der lange Nachmittag eines 
zweiten (bzw. dritten) Frühlings wird. Zum anderen vermag die wohlfahrtsstaatliche Wiederentdeckung des Alters, so deren leitende Idee, ,den Älteren genau jenen Anschluss an die Gesellschaft und damit genau jenen Lebenssinn wieder [zu] geben, der vielen von ihnen mit dem Eintritt in den Ruhestand abhanden kommt" (Münch 2009: 163) - ein sozialpolitisches Inklusionsmotiv, in dem, wie bei anderen Adressatengruppen produktivistischer Sozialpolitik auch, zugleich die bürgerliche Kontrollagenda einer „Motivierung zur normkonformen Teilnahme am gesellschaftlichen Leben“ (Münch 2009: 166) aufscheint.

Zwar muss an dieser Stelle die alters- bzw. altenpolitische Spezifik dieser „nachholenden“ Verbürgerlichung betont werden, denn anders als bei der Umgestaltung etwa des wohlfahrtsstaatlichen Sicherungsregimes für erwerbsfähige Erwerbslose spielen bei den sozialpolitischen Versuchen zur Mobilisierung der Produktivitätspotenziale des Alters im engeren Sinne herrschaftliche - zwangsförmige oder repressive - Regierungstechnologien zumindest einstweilen keine Rolle. Auch die materiellen Sicherungspositionen der aktuellen Ruhestandspopulation werden, im Gegensatz zu jenen der Erwerbslosen oder zukünftiger Rentner/innen, nicht angetastet, das ,produktive Alter“ soll sich im Kontext und auf der Grundlage bestehender Versorgungsansprüche entfalten. Doch erscheint die produktivistische Anrufung der Alten gerade vor dem Hintergrund ihrer wohlfahrtsstaatlich ,privilegierten“ Stellung als eine zwar wenig herrschaftsförmige, aber deswegen nicht weniger machtvolle gesellschaftspolitische Tendenz (van Dyk et al. 2010). Eine Tendenz, die in ihrer regulativen Idee - soziale Inklusion eines/r Jeden als produktives Subjekt der Bürgergesellschaft - auf die sozialstrukturellen Trägergruppen des Bruchs mit dem sozialpolitischen Konstrukt des „Ruhestands“ verweist: Es sind die Selbstverwirklichungs- wie Leistungswerte gleichermaßen vertretenden, auf individuelle Selbstbestimmung wie gesellschaftliche Teilhabe orientierten, „neubürgerlichen“ Post-68erMilieus (Siri 2010), die in bemerkenswerten Diskurskoalitionen gegen eine „fürsorgliche Vernachlässigung“" (Nolte 2004) der Sozialstaatsbürger/innen und für eine produktive Mobilmachung aller Sozialkategorien streiten - und die in diesem gesellschaftspolitischen Impetus auch die Alten und das Alter nicht vergessen.

Über die sozialen Effekte der angestrebten „Einbürgerung“ des Alters in eine produktivistische Bürgergesellschaft lassen sich einstweilen nur mehr oder minder begründete Vermutungen anstellen. Zum einen ist davon auszugehen, dass sich die Kluft zwischen dem „dritten“, ,jungen“ - und als solchem produktiv regierbaren - Alter einerseits, dem ,vierten“, abhängigen, pflegebedürftigen, somit „wirklich“ alten Alter andererseits - das sich im Sinne einer „produktiven“ Sozialpolitik als unregierbar erweist - tendenziell weiten wird, mit absehbar negativen Konsequenzen für die gesellschaftliche Legitimation „nicht-produktiver“ Lebenslagen. Zum anderen, und dieser Vermutung soll im Folgenden anhand empirischen Materials nachgegangen werden, dürften sich innerhalb der ,jungen Alten“" sozialstrukturelle Differenzierungslinien verstärken, die entlang der neuen bürgergesellschaftlichen Altersnorm verlaufen - stehen doch erhebliche symbolische Distinktionsgewinne für all jene Älteren zu erwarten, die milieubedingt den produktivistischen Verhaltensidealen ohne Weiteres nachkommen können oder ihnen in ihrer alltäglichen Lebensführung ohnehin bereits entsprechen. Dass die heutigen Alten ,im Durchschnitt gesünder, besser ausgebildet und vitaler [sind] als frühere Generationen“" (Deutscher Bundestag 2010: V; identisch: Deutscher Bundestag 2006: 4) ist ein sozialstatistischer Befund, der altenpolitisch offensichtlich zu dem Fehlschluss verleitet, jede/ r Alte könnte auf seine/ihre Weise den Normalitätsannahmen des produktiven Alter(n)s gerecht werden. Was zu beweisen wäre - bzw. zu widerlegen ist.

\section{Deutungsmuster subjektiven Alter(n)s}

Wie deuten ältere Menschen selbst das Alter(n), auf welche Konzepte vom „guten“ Alter(n) greifen sie zurück? Und inwiefern spielen sozialpolitische Konzepte wie das des produktiven 
Alter(n)s für sie überhaupt eine Rolle? Dieser Frage sind wir im Rahmen unserer qualitativen Studie nachgegangen. Dabei zielte unsere Forschung auch darauf ab, Tendenzen einer möglichen sozialstrukturellen Spezifik produktivitätsorientierter Alter(n)skonzepte auszumachen. Uns interessierte also auch die Frage, ob sich in unserem empirischen Material unterschiedliche Deutungsmuster zeigen, die sich als jeweils „typisch“ für Angehörige spezifischer Sozialmilieus verstehen lassen.

Empirische Untersuchungen subjektiver Alter(n)serfahrungen stehen prinzipiell vor dem Problem, dass Alter(n) eine äußerst uneindeutige soziale Erfahrung ist. Im Leben der Einzelnen ist es theoretisch zwar jeden einzelnen Tag präsent, erfahren wird es jedoch meist indirekt: Das Alter selbst ist kaum dingfest zu machen. Nicht zuletzt daraus erklärt sich das inzwischen vielfach bestätigte Phänomen der subjektiven „Alterslosigkeit“ alternder Menschen (vgl. Hockey / James 2003; Kaufmann 1986; Jones 2006). Auch der retrospektive und prospektive Blick auf den eigenen Alternsprozess artikuliert weniger das „tatsächliche“ Erleben des gegenwärtigen Älterwerdens als vielmehr eine durch die jeweils aktuelle Lebens- und damit Altersphase geprägte Sicht der Dinge (Biggs 2005: 120). Nichtsdestotrotz spielen Altersbilder - also Zuschreibungen von „erwünschten“ und „unerwünschten“, „eigentlichen“ und „uneigentlichen" Formen des Alter(n)s - in den Selbstdeutungen alternder Subjekte eine wichtige Rolle (Graefe / van Dyk 2012).

Alter(n) ist zugleich ein wichtiger Marker für soziale (Selbst-)Verortungen: Der gesellschaftlichen Kategorisierung nach Alter lässt sich kaum entkommen. Nicht nur die altersbezogene - und nach wie vor weitgehend gültige - Institutionalisierung von Lebensläufen (Kohli 1985) objektiviert die soziale Klassifikation nach Alter. Auch habituelle Praxen sind stets und ständig altersbezogen - ,regardless of whether we are conscious of our age and how we categorize ourselves, in interaction and various institutional practices we are expected to perform age and behave age-appropriately“ (Pietilä / Ojala 2011:381). Soziale Zugehörigkeiten werden subjektiv jedoch nicht nur affirmativ, sondern auch durch - verbale, emotionale und soziale Distanzierung stabilisiert. Dies gilt für Klassenmilieus (Bourdieu 1982) ebenso wie fürs Alter(n): Subjektive Vorstellungen vom ,guten” Alter(n) implizieren immer auch mehr oder minder explizite Vorstellungen eines ,schlechten” und in diesem Sinne unerwünschten bzw. abgelehnten und abgewehrten Alter(n)s. Zu vermuten ist, dass klassen- und alter(n)sspezifische Formen sozialer Distinktion nicht nur nebeneinander existieren, sondern in spezifischer Weise interagieren. Dieser Zusammenhang gewinnt mit der Durchsetzung des Leitbildes vom produktiven Alter(n) besondere Brisanz, reflektiert dieses doch nicht nur eine „zunehmende Dominanz von Normen des mittleren Lebensalters" und in diesem Sinne einen sozialpolitischdiskursiven „middle-ageism“ (van Dyk / Lessenich 2009: 38), sondern trägt darüber hinaus potenziell zu einer Stabilisierung bürgerlich-mittelschichtiger Verhaltensnormen und Lebensstile bei.

\subsection{Ergebnisse der empirischen Studie}

Eben dies soll im Weiteren auf der Grundlage unserer Forschungsergebnisse genauer untersucht werden. In unserem Material fanden wir vor allem zwei zentrale und kontrastierende Deutungsmuster des Alter(n)s: Subjektives Alter(n) wird einerseits im Hinblick auf die Frage der biografischen (Weiter-)Entwicklung, der individuellen Wandlungsfähigkeit und der sinnvollen Aktivitäten thematisiert. Demgegenüber wird es andererseits - von anderen Befragten - vorrangig im Hinblick auf die Kontinuität alltäglicher Lebensführung thematisiert und auf diese Weise weniger biografisch, als vielmehr gegenwartsbezogen gedeutet. Im Folgenden werden diese zwei zentralen Deutungsmuster des Alter(n)s jeweils entlang drei verschiedener Topoi (,gutes Altern / schlechtes Altern“, „Aktivität und Engagement“, „Übergänge zum Alter") kontrastiert (3.1.1 und 3.1.2) und zusammenfassend gegenübergestellt sowie unter der 
Fragestellung diskutiert, inwiefern sich die kontrastierenden Deutungsmuster als sozialstrukturelle, Typiken“ interpretieren lassen (3.2). Im Anschluss daran skizzieren wir weiterführende Überlegungen zum Zusammenhang von (produktivem) Alter(n), Geschlecht und Klassenmilieus (4).

\subsubsection{Alter(n) als (weitere) Gelegenheit zur Wandlung und Entwicklung}

Im Rahmen dieses Deutungsmusters stellt sich das ganze Leben als Lernprozess dar - auch das Alter(n), das gleichsam besondere Chancen und Herausforderungen für die eigene Weiterentwicklung bietet. „, Gutes Alter(n) “ heißt dementsprechend, das Alter in eine Lerngelegenheit zu verwandeln. Maßgeblich dabei ist die Annahme, dass es vor allem die innere Einstellung zum Alter(n) ist, die über dessen Qualität entscheidet. Diese innere Einstellung aber ist keine fixe Haltung oder eine ein für allemal erworbene Eigenschaft - sie muss selbst laufend gepflegt und verbessert werden. So formuliert etwa Frau Beck, 62 Jahre alt und Stadtplanerin im Vorruhestand, regelrecht ein Programm fürs optimale Älterwerden: ,[...] mindestens einmal im Jahr sich mit diesem Thema auseinanderzusetzen, spätestens am Geburtstag. Und vorbauen. Das heißt, moralische Werte, an denen man sich orientiert, immer wieder überprüfen [...]. Seine Fähigkeiten immer wieder neu ausbauen, testen, über den Rand hinaus leben, denken, und offen sein für andere Dinge“. ${ }^{5}$ Auch die 72 -jährige Frau Meyerhoff, ehemalige Sozialarbeiterin, betont, dass ihr ihre optimistische Grundhaltung, von der sie jetzt im Alter profitiert, nicht in den Schoß gefallen sei. Es war „große Arbeit dahin zu kommen. Zu dieser Lebenseinstellung halt".

Distanz gegenüber den Lebensweisen anderer Älterer wird dabei durchaus auch formuliert. Ein wiederkehrendes Motiv ist hier die Kritik eines übertriebenen Materialismus - gegenüber dem „Hängen am Materiellen“ bzw. ,hirnlosem Konsumverhalten“. Ein weiteres Motiv betrifft die Einstellung zum Alter. Herr Münstermann etwa, 76-jähriger ehemaliger Pfarrer, hält eine „rückwärtsgewandte Haltung im Alter“ für problematisch. Mit dem vielen Jammern täten „sich Menschen also nichts Gutes" - und das, wo sie doch, wie er am Beispiel zweier Bekannter ausführt, „,eigentlich anders leben „,[könnten], wenn sie das Ding bissel rumdrehen“, das heißt hier: sich eine veränderte, positivere Sicht auf das Leben zulegen würden. Empathie und Abgrenzung, Verständnis (für schwierige, , unverschuldete“ Lebenssituationen) und Unverständnis (für rückwärtsgewandte oder unnötig negative Einstellungen) gehen hier Hand in Hand.

Aktivität ist ein zentraler Topos in allen im Rahmen unserer Studie geführten Interviews. Die Frage, ob und wie aktiv man im Alter ist bzw. sein will, spielt eine wichtige Rolle für die subjektive Wahrnehmung und praktische Gestaltung des Alltags. Fast ebenso durchgängig finden sich Negativbewertungen von „Nichtstun“ im Alter. Dabei differiert, welche Bedeutung den jeweils genannten Aktivitäten zugeschrieben wird: Wird Alter(n) als Zeit der biografischen Reifung bzw. Wandlung verstanden, muss Aktivität dem Anspruch an Sinnerfüllung genügen. Es geht nicht darum, irgendetwas, sondern darum, Sinnvolles zu tun. So erklärt etwa Frau Schwarz, 49-jährige Projektmanagerin, dass sie am liebsten jetzt schon in Rente ginge: ,[...] aber nicht, weil ich gern zuhause auf dem Sofa liegen würde, sondern weil ich denke, dass so viel zu tun wäre [...] also ich würd mich viel mehr gerne gesellschaftspolitisch engagieren, ich wäre gerne Leseoma in der Kita“. Unsere Gesprächspartner/innen entwerfen ein breites Panorama unterschiedlichster nacherwerblicher Tätigkeiten: familiäres und soziales Engagement, Reisen, Fremdsprachen, Nachbarschaftshilfe, vielfältige kulturelle Aktivitäten etc. Be-

5 Wir verzichten hier und im Folgenden, im Interesse besserer Lesbarkeit, jeweils auf die genaue Dokumentation der Textstellen in den Interviews (in Form von Nummer- und Zeilenangaben). Die Namen der Befragten sind selbstverständlich anonymisiert. 
tont dabei wird aber nicht so sehr die bloße Fülle, sondern der Aspekt des lebenslangen Lernens und der persönlichen Weiterentwicklung. Lernen und Entwicklung werden dabei nicht als Spezifika des Alters verstanden, sondern als biografische Konstanten, die im Alter aufgrund der größeren Zeitressourcen jedoch (noch) besser zu realisieren sind. Frau Leitmeyer, 66jährige Architektin, die ehrenamtlich in mehreren Bürgerinitiativen aktiv ist, beschreibt als Gewinn im Alter, dass sie nun ohne Einschränkungen ihren ,normalen Leidenschaften“ frönen kann: Malen, Lesen, Klavierspielen.

Alter(n) als Gelegenheit zur Wandlung zu verstehen impliziert die Überzeugung, dass sinnvoll verbrachte Zeit die Grundlage einer erstrebenswerten Lebensgestaltung ist. „Sinn“ stiftet dabei das, was eine bislang existierende Konstellation - sei diese individuell, intersubjektiv oder gesellschaftlich gerahmt - verändert. Aktivität steht somit im Dienste der Weitentwicklung einer gegebenen Situation, kontinuierlicher Erwerb von Wissen ist dafür zentral. Selbstverständlich gibt es in diesem Prozess des (Selbst-)Wandels auch Ruhepausen; doch auch diesen wohnt ein tieferer Sinn inne. Frau Meyerhoff etwa unterscheidet genau zwischen negativem „Lahmsein“ und positiver „Geruhsamkeit“, wobei sie sich Letzteres mit zunehmendem Alter mehr als früher zugesteht. In Geltung bleibt jedoch auch dabei das Modell der Entwicklung: Etwas kürzer zu treten ist eine Folge der Einsicht, dass man auch an Aktivitäten „fasten“ sollte, also „sieben Wochen mal geruhsam leben“; man „darf“ pausieren, sofern die Pausen der ,inneren Reinigung“ zuarbeiten.

Alter(n) als Gelegenheit zur Wandlung zu verstehen impliziert zugleich, dem Erleben und der Gestaltung biografischer Übergänge eine wichtige Rolle in Bezug auf Alter(n) und Selbstkonzept zuzuschreiben. Dies wird beispielhaft in der Thematisierung des 50. Geburtstags deutlich. Ganz offensichtlich fungiert dieser Tag allgemein als Datum, an dem sich eine Problematisierung oder zumindest Zurkenntnisnahme des eigenen Älterwerdens aufdrängt. Solcherart zum Thema geronnen, kann es dann bewusst bearbeitet oder auch ausdrücklich für weniger wichtig befunden werden (vgl. Nikander 2009). Gesprächspartner/innen, die Alter(n) als Gelegenheit und Aufgabe zur Entwicklung und Wandlung rahmen, thematisieren Übergänge wie den 50. Geburtstag (prospektiv wie retrospektiv) ausführlich. Geburtstage werden hier oft von - auch selbst kreierten - Ritualen begleitet. Es sind also nicht allein die Aktivitäten und das eigene Selbst, die bewusst ,gestaltet“ werden, sondern auch der individuelle Lebenslauf. Nicht nur die Alltagszeit des täglichen Lebens, auch die Lebenszeit der gesamten Biografie (vgl. Alheit 1988) bildet einen wichtigen Bezugspunkt der Selbstregulation und -konstruktion. Dies gilt nicht zuletzt für den institutionalisierten Übergang vom sogenannten „Zweiten“ ins „dritte“ Lebensalter, den Renteneintritt: Er wird hier vor allem als Chance zur individuellen Neuorientierung und Weiterentwicklung thematisiert, bisweilen auch bewusst ritualisiert.

Teilweise erstreckt sich die entsprechende Deutung sogar auf den Übergang ins sogenannte „vierte“, hohe, pflegebedürftige Alter. Zwar wird dieser Übergang in unseren Interviews durchgängig eher wenig und wenn, dann häufig im Modus von Negation thematisiert - etwas, das man nicht erleben und sich eigentlich auch nicht näher vorstellen möchte (Graefe et al. 2011). Es überwiegen Formulierungen wie ,so lange so weitermachen, wie es noch geht“, „wenn es dann mal vorbei ist“ oder ,darüber denke ich bewusst nicht nach“. Allerdings finden sich auch Skizzen eines hohen Alters als weitere - und durchaus aussichtsreiche - Etappe der biografischen Entwicklung. Frau Schwarz etwa betont, sie freue sich regelrecht auf die Hochaltrigkeit. Aus ihrer Sicht handele es sich um eine Lebensphase, in der man seinen ,inneren Reichtum“ entdeckt - ,und das entdeckt man natürlich besser, wenn man älter ist als wenn man jünger ist". Andererseits erklärt etwa Herr Münstermann, die Aussicht auf eine letzte Lebensphase in Pflegeheim und Rollstuhl mache ihm ,überhaupt nix aus“, im Gegenteil: ,[...] es kommt die Phase des Abschiednehmens oder des Gehens [...], die Phase, wo ich nicht mehr 
gestalten kann und auch nicht mehr muss. Und dann möchte ich das [...]“. Das für dieses Deutungsmuster zentrale Primat der Gestaltbarkeit des eigenen Lebens(laufs) kommt hier paradoxerweise auch dort noch zur Anwendung, wo alle Gestaltungsambition versagen muss: Wenn nichts mehr geht, ist auch dies letztlich ,selbst gewollt“.

\subsubsection{Alter(n) als Kontinuität alltäglicher Lebensführung}

Wird Alter(n) demgegenüber als Fortsetzung und Weiterführung des „normalen“ Lebens verstanden, so geht man davon aus, dass sich im Alter nichts Wesentliches gegenüber früheren Lebensphasen verändert. Es geht also gerade nicht darum, sich permanent zu wandeln und zu entwickeln, sondern darum, sich auch im Alter möglichst gleich zu bleiben - und unvermeidliche Veränderungen, wie etwa die Verrentung oder gesundheitliche Einschränkungen, in das Kontinuum der Lebensführung einzupassen. Zentral für dieses Deutungsmuster des Alter(n)s ist weniger die Institution des „Selbst“" als vielmehr die alltäglich praktizierte Lebensführung. Altersbedingte Veränderungen erscheinen als ,äußere“ Veränderungen, die möglichst störungsfrei zu bewältigen sind. So erklärt etwa die 93-jährige FrauHorten, Hausfrau: ,[...] des [Alter] is nix anderes wie das Leben auch bisher. Ja sicher ist es anders, wenn Sie jetzt, äh ich freu mich genauso am Leben, aber es ist eben des es ändert ja nichts in meinen, in meinen Auffassungen, in meinem Empfinden, es ändert nur, dass ich nimmer alles kann“.

Im Rahmen dieses Deutungsmusters wird Alter(n) - und spezifischer , gutes Alter(n) “konzipiert als Kontinuität des Lebens unter mehr oder minder veränderten materialen und organisatorischen Bedingungen, und das heißt auch: Je weniger sich ändert, umso „,besser“ das Alter. Zwar thematisieren die Gesprächspartner/innen hier natürlich auch Veränderungen im Zusammenhang mit dem Prozess des Älterwerdens, dabei ausdrücklich auch positive, doch werden diese nicht unbedingt als Gelegenheit zur Reifung und zum persönlichen Wachstum begriffen. Ein Beispiel dafür ist der Aspekt der Zeitgestaltung. Nachberuflich „mehr Zeit“ für sich zur Verfügung zu haben und über ihre Gestaltung selbstbestimmt entscheiden zu können, wird fast durchgängig positiv bewertet, nicht jedoch als „Lerngelegenheit“" gerahmt. Für Herrn Granauer etwa, 68 Jahre alt und ehemaliger Verwaltungsangestellter, ist positiv am Alter, dass „,man kein Stress in dem Sinne [hat], beruflich, man hat mehr Zeit für sich, man sieht die Enkelkinder [...] ranwachsen und ich bin mein eigener Herr". Gutes Alter(n) zeichnet sich hier eher durch die Abwesenheit von Fremdbestimmung aus sowie durch die Fähigkeit, altersbedingte Einschränkungen so zu kompensieren, dass die eingeübte Alltagsgestaltung aufrechterhalten werden kann.

Abgrenzungen gegenüber dem „schlechten“ Alter(n) Anderer werden hier ebenfalls vorgenommen. Dabei steht die Klage über die Verletzung von Reziprozitätsnormen im Vordergrund; Unverständnis wird zudem über mangelnden Familienzusammenhalt sowie Faulheit und Passivität anderer Älterer geäußert. Die Kritik richtet sich meist auf konkrete Andere in mittlerer Distanz - Nachbar/innen, die mit ihrer Familie nichts unternehmen, Bekannte, die nicht aus dem Haus gehen, oder solche, die sich für einen erwiesenen Gefallen nicht dankbar zeigen. Negativ wird außerdem bewertet, wer anscheinend mit seiner Zeit nicht umzugehen versteht, sich nicht zu beschäftigen weiß. So erklärt Frau Brandt, 64jährige Sekretärin im Ruhestand: „Ja, also ich find's schlimm eigentlich, wenn man älter wird und nicht weiß, was man mit seiner Zeit anfangen soll, also so in der Nachbarschaft".

Aktivitäten spielen demnach auch hier eine zentrale Rolle. Im Vordergrund steht dabei jedoch nicht der Aspekt des Lernens oder der Selbstentwicklung. Frau Brandt etwa nutzt gerne das städtisch subventionierte Kinoprogramm für Über-50-Jährige - und zwar ,weil's günstiger ist“. Den Kinobesuch verbindet sie mit einem Stadtbummel, dann ,ist der ganze Tag schon mal ausgefüllt"“. Zeit scheint hier etwas zu sein, das reichlich vorhanden ist und mit Aktivität 
verbracht - und verbraucht - wird. Der 67-jährige Herr Hansen, ehemaliger Krankenpfleger, verbringt seine Tage als Hausmann, Hundehalter und mit ehrenamtlichen Tätigkeiten. Anders als Frau Meyerhoff oder Frau Schwarz aber betont er jedoch weniger stark, wie voll seine Tage sind und wie knapp seine Zeit ist. Er ist eben „,beschäftigt - und wenn ich mal Langeweile hab mach ich Augenpflege, ne“. Pausen und Langeweile erscheinen hier weniger als Lektion in „Geruhsamkeit“, denn als Zeitfenster, die aktiv zu füllen sind - oder eben auch nicht.

Aktivität kann und sollte - so jedenfalls sieht es das Leitbild vom produktiven Alter(n) vor - auch soziales Engagement umfassen. Während diese Vorstellung von den „,wandlungsorientierten" Befragten in der Regel prinzipiell begrüßt (und oft, wenngleich nicht immer, auch umgesetzt) wird, berichten ,kontinuitätsorientierte“ Befragte oft erst auf Nachfrage von ihrem - durchaus auch praktizierten - ehrenamtlichen Engagement; es erscheint hier eher als eine mögliche Tätigkeit unter vielen anderen und nicht so sehr als besondere Gelegenheit individueller und gesellschaftlicher „Sinnstiftung“. Bisweilen wird ehrenamtliches Engagement auch ausdrücklich negiert und gegen die eigene selbstbestimmte Lebensgestaltung abgewogen. Frau Brandt etwa erklärt auf die Frage nach einem eventuellen sozialen Engagement, dass sie daran nicht interessiert sei: „[...] dann müsste ich jetzt mein eigenes Leben einschränken, also ich könnte nicht mehr so frei planen wie wir's jetzt im Moment tun“".

Gesprächspartner/innen, die Alter(n) vor allem unter dem Aspekt der Kontinuität der Lebensführung beschreiben, berichten kaum von ritualisierten biografischen Übergängen. Dies gilt auch für den klassischen Altersübergangsmarker 50. Geburtstag: Er liefert keinen Anlass für eine reflektierende Neujustierung des Selbstkonzeptes. Auch der Übergang in den Ruhestand wird weniger als Eintritt in eine neue biografische Phase interpretiert, denn als Ende des Berufslebens, woraus sich neue Anforderungen und Chancen der Alltagsorganisation ergeben. Deutlicher als im erstgenannten Deutungsmuster wird hier zudem die Frage der finanziellen Absicherung thematisiert. Eine auskömmliche Altersrente erscheint weniger als notwendige Basis für sinnvolle Aktivität und in diesem Sinne als Mittel zum Zweck, sondern eher als Zweck an sich. Deutlich formuliert dies etwa Herr Hansen, wenn er feststellt: „Ja so bin ich immer zeitlebens so mein ganzes Leben gegangen, immer in Gedanken dran, Rente, Altwerden und äh naja bisschen aufsteigen, was mir dann auch gelungen ist, dass ich auch ne gute Rente habe, ne?" Die Rentenhöhe gerät hier zum Indiz für den gelungenen sozialen Aufstieg, die nachberufliche Zeitautonomie erscheint als angemessene Konsequenz einer lebenslangen beruflichen Aktivität.

In Bezug auf den Übergang ins sogenannte „vierte“ Lebensalter gilt hier zunächst, wie im Falle der „wandlungsorientierten“ Befragten auch, dass dieser eher wenig und, falls doch, zumeist vage thematisiert wird. Anders als im Zusammenhang mit dem erstgenannten Deutungsmuster finden sich hier jedoch keine Vorstellungen vom hohen und pflegebedürftigen Alter als letzte biografische Etappe, in der wahlweise „Annehmen“ gelernt oder ,innerer Reichtum" entdeckt werden kann. Vielmehr erscheint das hohe und abhängige Alter, dort, wo es expliziter thematisiert wird, bisweilen schlicht als Alptraum. So erklärt etwa Herr Hansen, er „,möchte nicht als Tattergreis dann irgendwo rumgeifern, gell““. Auch Herr Granauer betont in Bezug auf die von ihm antizipierte Zukunft: , [...] wir machen unsern Trott weiter, wird sich net viel ändern, wenn wir net, wovor wir beide Angst haben, ins Altersheim müssen oder verblöden oder sonst was“. Wo die Kontinuität der Lebensführung bedroht scheint, gerät Alter zum Unbewältigbaren schlechthin: Man kann es nicht gestalten, sondern bloß fürchten.

\subsection{Zusammenfassende Kontrastierung}

Stellt man die beiden hier skizzierten Deutungsmuster gegenüber, so lassen sie sich idealtypisch zwei gegenteiligen Modellen oder „Strategien“ im Umgang mit dem Alter(n) zuordnen: Alter(n) wird im einen Fall durch eine lebenslange Arbeit am „wandlungsfähigen Selbst“, im 
anderen durch Bewahrung und Anpassung bewährter Lebensführungsmuster bewältigt. Interessanterweise führen beide Strategien dazu, dass sich die meisten der Befragten subjektiv relativ ,alterslos“ fühlen: Sie berichten entweder von einer erheblichen Differenz zwischen objektivem und gefühltem Alter und/oder betonen, dass das Älterwerden „eigentlich“ für sie keine wesentliche Bedeutung hat (vgl. Kolland 2010: 77).

Das Modell des „wandlungsfähigen Altersselbst“ zielt darauf, das eigene Alter(n) als Entwicklungschance zu begreifen. Die Maxime lebenslanger Veränderung stellt dabei das Leitmotiv dar. Die gleichzeitige Annahme eines zwar lebenslang zu bearbeitenden, aber eben darin kontinuierlichen Selbst ermöglicht es, das Älterwerden als relativ „äußerliche“ Erfahrung zu konzipieren, die das eigentlich alterslose Selbst nicht grundsätzlich erschüttert. Im Modell des „kontinuitätsorientierten Alter(n)s“ hingegen resultiert die subjektive Alterslosigkeit aus der Wahrnehmung einer relativ stabilen Kontinuität von Alltagspraktiken und sozialen Beziehungsnetzen. Hier begründet weniger die Kontinuität des alterslosen Selbst als vielmehr die Stabilität der alltäglichen Bedürfnisse und Notwendigkeiten die Erfahrung relativer Alterslosigkeit.

Neben den dargestellten Differenzen in Bezug auf die Wahrnehmung und Bewertung des Alter(n)s verweist dieser Kontrast zugleich auf unterschiedliche Konzepte von Subjektivität. Im erstgenannten Modell entwirft sich das (alternde) Individuum zugleich als Subjekt und Objekt der eigenen Biografie: sie wird nicht nur erlebt, sondern zugleich bearbeitet. Vom deutenden Subjekt wird ein ,Selbst" unterschieden, das entwickelt und - bestenfalls - auch biografisch „optimiert“" werden kann. Nicht nur der Körper, auch das „Innere“ - die Einstellungen und Gefühle - erscheinen dabei als gestalt- und veränderbar. Im zweitgenannten Modell geht es zwar auch um Gestaltbarkeit. Gestaltet aber werden soll weniger das eigene „Selbst“, als vielmehr das alltägliche Leben - und diese Gestaltung gelingt, je nach materiellen und sozialen Bedingungen, besser oder schlechter.

Aktivität ist dabei in beiden Modellen wichtig, wird jedoch unterschiedlich konzipiert: als wandlungsorientierte (Lebens-)Aufgabe oder als auf aktuelle (Alltags-)Aufgaben bezogenes Beschäftigtsein. Aktivitäten, die auf Entwicklung und Erfüllung hinzielen, orientieren sich notwendig an der Zukunft. „Beschäftigtsein“ hat demgegenüber seinen Zweck eher in sich selbst und vorrangig einen Gegenwartsbezug: die Zeit ist verbracht, der Tag vorbei. Im ersten Fall handelt es sich um eine Logik der Investition, im zweiten um eine des Ge- und Verbrauchs von Zeit.

Entsprechend werden auch Altersübergänge unterschiedlich interpretiert: Während ihre rituelle Gestaltung im Konzept des ,wandlungsfähigen Altersselbst“ eine zentrale Rolle spielt, sind sie für das Modell des „kontinuitätsorientierten Alter(n)s“ eher von untergeordneter Bedeutung. In Bezug auf das hohe, pflegebedürftige, ,vierte“ Alter liegt die Differenz zwischen beiden Deutungsmustern nur vordergründig im Unterschied zwischen Auf- und Abwertung des hohen Alters. Letztlich verweisen die Befunde auf unterschiedliche Konzeptionen der eigenen Biografie bzw. Lebenszeit: Ist die gesamte Biografie ein Projekt zur Entfaltung und Verbesserung des Selbst, dann schließt dies das hohe Alter mit ein - Lerngelegenheiten hält auch dieses bereit. Insofern die lebenslange Arbeit am Selbst notwendig selbstreflexiv ist, bedroht erst der Eintritt einer Demenzerkrankung, nicht aber möglicher körperlicher Verfall diese Selbstkonzeption. Wird das Alter andererseits als Lebensphase konzipiert, bei der es darauf ankommt, das eigene Leben so störungsfrei wie möglich ,,am Laufen“ zu halten, dann signalisiert bereits der Eintritt von Pflegebedürftigkeit ein bedrohliches Scheitern dieses Konzeptes.

Selbstverständlich finden sich in fast allen unserer Interviews auch Inkonsistenzen sowie Mischformen zwischen beiden hier skizzierten Modellen; es handelt sich weniger um ge- 
schlossene Konzepte als vielmehr um perspektivische Tendenzen, um - je nach Gegenstand mehr oder weniger fluide - Orientierungsrahmen, die sich im Material in je unterschiedlicher Explizität und Intensität zeigen (vgl. Amrhein / Backes 2008: 388). Es lassen sich auf Basis unserer Interviews somit keine altersspezifischen „Identitäten“ konstruieren. Eine ausdifferenzierte Milieu- bzw. altersbezogene Lebensstilanalyse (vgl. Wahl 2003) ist auf der Grundlage eines zahlenmäßig begrenzten qualitativen Samples zudem nicht möglich. Geht man allerdings vom deutlichsten Kontrast der hier untersuchten alter(n)sbezogenen Deutungsmuster aus, so ergibt sich eine erkennbare Differenz in Bezug auf die Milieu- und die Geschlechterdimension: Das Modell des „wandlungsorientierten Altersselbst“ finden wir ausgeprägt vor allem bei Frauen mit akademischer Bildung. ${ }^{6}$ Das Modell des „kontinuierlichen Alter(n)s“ findet sich hingegen vor allem bei Befragten (Männern wie Frauen) mit mittleren und niedrigen Bildungsabschlüssen. Zwar können wir auf Basis einer Querschnittsstudie in Bezug auf die hier interessierende subjektiv vermittelte symbolisch-normative Dimension nicht konstatieren, was für objektiv ungleiche Lebenslagen gilt - dass im Alter nämlich „,der Schichteinfluss [...] gleichförmig erhalten“ bleibt (Kohli et al. 2005: 329). Insofern aber die hier diskutierten zwei zentralen kontrastierenden Deutungsmuster jeweils sowohl von jüngeren als auch von älteren Gesprächspartner/innen artikuliert werden, können wir vermuten, dass sie sich in ihrer Grundstruktur (Wandlungs- bzw. Kontinuitätsorientierung) zumindest in der zweiten Lebenshälfte als relativ stabil erweisen.

Zusammenfassend lässt sich festhalten, dass das „,wandlungsorientierte Altersselbst“ vor allem um den Topos der Individualität, das Modell des „kontinuitätsorientierten Alter(n)s“ hingegen um jenen der Normalität organisiert ist. Die Behauptung, ,individuality operates as an unequal resource“" (Skeggs 2004: 55), lässt sich somit auf Basis unseres Materials plausibilisieren: Die Idee, ein vom Subjekt konzeptionell unterscheidbares, gestalt- und vor allem auch optimierbares „Selbst“ zu haben, ist gesellschaftlich offenbar ungleich verteilt, genauer gesagt: „The self is a classed term that produces difference through its utterance“ (Skeggs 2004: 134). In der Tendenz jedenfalls, darauf deuten unsere Ergebnisse hin, weist das Konzept vom gestaltbaren Selbst eine deutliche Affinität zu den Lebensstilen der gehobenen Bildungsmilieus auf und ist zudem geschlechtsspezifisch markiert (Graefe / van Dyk 2012). Eine Erklärung für Letzteres könnte sein, dass das „Selbst“ traditionell eher in der Sphäre der Gefühle und damit auch des Privaten angesiedelt, somit konzeptionell eher „weiblich“ ist. Anzunehmen wäre dann, dass sich die Geschlechterdifferenz mit der (wenigstens diskursiven) Aufweichung traditioneller Geschlechterrollen in zukünftigen älteren Generationen verringern wird.

\section{Ausblick und Diskussion}

In welchem Zusammenhang stehen die hier rekonstruierten empirischen Befunde nun zu dem einleitend skizzierten Phänomen einer Anrufung des ,produktiven Alters“ im Kontext des gegenwärtigen Wandels zum ,aktivierenden Sozialstaat"? Zumindest tentativ lässt sich annehmen, dass das Modell des „wandlungsorientierten Altersselbst“, in diesem Lichte gesehen, sich in mehrerlei Hinsicht kompatibler und anschlussfähiger erweist als jenes des „kontinuitätsorientierten Alter(n)s“: Zum einen, insofern Ersteres um den Topos der „selbstverantwortlichen und sinnstiftenden Aktivität“ zentriert ist, für den der wohfahrtsstaatliche Fokus auf die „ressourcenstarken Alten“ eine wesentliche Grundlage bildet. Zum anderen, weil sowohl dem produktiven Alter(n) als auch dem ,wandlungsorientierten Altersselbst“ die Idee der permanenten Entwicklung und Optimierung inhärent ist - und zwar als Selbstzweck, nicht als Mittel

6 Dabei wäre es insbesondere bei weiblichen Befragten älterer Kohorten kurzschlüssig, von der Abwesenheit eines akademischen Abschlusses auf eine Nicht-Zugehörigkeit zu statushöheren Milieus zu schließen; hier sind vielmehr die soziale Herkunft und (ggf.) der Beruf des Ehemannes mit zu berücksichtigen. 
zu einer näher spezifizierten Zielerreichung. Drittens und vor allem aber, weil beide Konzepte die Differenz zwischen Individuum und Gesellschaft in der Konstruktion einer ,win-win“Konstellation tendenziell aufzulösen scheinen: Gesellschaftliche Anforderungen - an lebenslanges Lernen, bürgerschaftliches Engagement, körperliche und geistige Fitness - transformieren sich hier in „ureigene“ Wünsche und Ziele des selbstverantwortlichen Subjekts.

Die Frage, ob das Leitbild vom produktiven Alter(n) einen spezifischen Klassen-Bias hat, lässt sich somit - auf Basis unserer Ergebnisse - tendenziell bejahen. Präziser lässt sich formulieren, dass dieses Leitbild strukturell eher bürgerlich-mittelschichtigen Rechtfertigungsordnungen zuarbeitet - und den Angehörigen entsprechender Sozialmilieus neue Optionen der Generierung symbolischer Macht und sozialen Kapitals verspricht. Die Figur der ,jungen“, ressourcenstarken, potenzialreichen Alten ,„übersetzt “ und überführt das ur-bürgerliche Ideal des eigenverantwortlichen, gestaltbaren Selbst - jedenfalls theoretisch - ins (höhere) Alter. Damit orientiert sie sich zugleich - auf scheinbar paradoxe Weise - an Normen der mittleren Lebensphase: Produktivität und biografische Entwicklung, üblicherweise als Aufgaben des mittleren Lebensalters verstanden, werden zur lebenslangen Anforderung. In der Anrufung des produktiven Alter(n)s wird das Alter gesellschaftlich sichtbarer und - nämlich in seiner Differenz zu anderen Lebensphasen - unsichtbarer zugleich. Die damit einhergehende doppelte Orientierung auf die Lebensweisen und Lebensstile der „Mitte“ - der bildungsbürgerlichen Mittelschichten einerseits, der middle agers andererseits - führt allerdings auch dazu, so jedenfalls unsere Vermutung, dass das Alter zukünftig mehr als bislang zum Feld von gesellschaftlichen Anerkennungs- und Verteilungskämpfen werden wird, ein zunehmend bedeutsames Feld der sozialen Distinktion.

Der Wohlfahrtsstaat als Instanz der Verteilung gesellschaftlicher Lebenschancen und der Zuweisung sozialer Statuspositionen spielt für die Struktur und die Dynamik dieser Kämpfe eine entscheidende Rolle: Er kann sie zu moderieren und entschärfen suchen - oder aber sie reproduzieren und verstärken bzw., mehr noch, in ihnen Partei ergreifen. ${ }^{7}$ Gegenwärtig erscheint es - in Anbetracht seiner Geschichte als Projekt und Objekt kultureller Hegemonieproduktion nicht ganz zufällig - eher so zu sein, als würden seine Institutionen und Interventionen darauf zielen oder jedenfalls hinauslaufen, Letzteres zu tun. Je stärker das Leitbild des produktiven Alter(n)s die individuellen Entwicklungschancen betont - und die persönliche „Verantwortung“ für deren Realisierung -, umso wirksamer stabilisiert es seinen hier beschriebenen Klassencharakter. Je massiver in seinem Namen hingegen Möglichkeiten einer sozialpolitischen „Verpflichtung“ zum ressourcenangemessenen Altern ventiliert werden, umso unattraktiver wird es für die - unbedingt auf Selbstgestaltung setzenden - bildungsnäheren Milieus, und zugleich umso suspekter für die - amtlichen Aktivitätsaufforderungen gegenüber typischerweise skeptischen - unterprivilegierten Schichten.

Indem der offizielle sozialpolitische Diskurs zum ,produktiven Alter(n)“, zumindest bislang noch und einstweilen, auf die „Verantwortungs“-Karte setzt (Lessenich 2009 b), betreibt er eine subtile Klassenpolitik für das Alter - jene Lebensphase, in der zu Zeiten des wohlfahrtsstaatlichen „Ruhestandsmodells“ der gesellschaftliche Klassenkonflikt vorübergehend stillgestellt schien. Diese Klassenpolitik hat zwei Fronten: Sie trennt nicht nur die ,jungen“, ,,produktiven" Alten von den (so das Bild) ressourcenarmen und früh gealterten Alten aus der Unterschicht, sondern differenziert auch innerhalb der amorphen gesellschaftlichen „Mitte“ zwischen statusniedrigeren, eher kleinbürgerlichen Milieus, die mit der Vorstellung wandlungsorientierter Arbeit am Selbst wenig anfangen können, von jenen bildungsbürgerlichen, akademisch orientierten Schichten, denen das ,produktive Alter(n)“ die gern genutzte Chance

7 „Although there is no need to adopt a state-centered“ approach to class, it would seem to be an important improvement to move beyond stateless theories of the middle class." (Wacquant 1991, S. 58; vgl. Bourdieu 1987.) 
auf sozialen Distinktionsgewinn bietet. Auf diese Weise entsteht eine neue Rechtfertigungsordnung des Alter(n)s - in der die Struktur symbolisch und normativ vermittelter sozialer Ungleichheit freilich tendenziell beim Alten bleibt.

\section{Literatur}

Achinger, Hans (1958): Sozialpolitik als Gesellschaftspolitik. Von der Arbeiterfrage zum Wohlfahrtsstaat, Reinbek.

Alheit, Peter (1988): Alltagszeit und Lebenszeit, in: Rainer Zoll (Hrsg.), Zerstörung und Wiederaneignung von Zeit, Frankfurt / Main, S. 371-386.

Amann, Anton / Franz Kolland (2008): Kritische Sozialgerontologie - Konzeptionen und Aufgaben, in: Dies. (Hrsg.), Das erzwungene Paradies des Alters? Fragen an eine kritische Gerontologie, Wiesbaden, S. 13-43.

Amrhein, Ludwig (2008): Drehbücher des Alter(n)s. Die soziale Konstruktion von Modellen und Formen der Lebensführung und -stilisierung älterer Menschen, Wiesbaden.

Amrhein, Ludwig / Gertrud M. Backes (2008): Alter(n) und Identitätsentwicklung. Formen des Umgangs mit dem eigenen Älterwerden, in: Zeitschrift für Gerontologie und Geriatrie 41, S. 382-393.

Backes, Gertrud M. / Wolfgang Clemens / Klaus R. Schroeter (2001): Zur Konstruktion sozialer Ordnungen des Alter(n)s, in: Dies. (Hrsg.), Zur Konstruktion sozialer Ordnungen des Alter(n)s, Opladen, S. 7-29.

Biggs, Simon (2005): Beyond Appearances: Perspectives on Identity in Later Life and Some Implications for Method, in: Journal of Gerontology: Social Sciences 60B, S. 118-128.

Bourdieu, Pierre (1982): Die feinen Unterschiede. Kritik der gesellschaftlichen Urteilskraft, Frankfurt / Main.

Bourdieu, Pierre (1987): What Makes a Class? On the Theoretical and Practical Existence of Groups, in: Berkeley Journal of Sociology 32, S. 1-18.

Bourdieu, Pierre (1998): Staatsgeist. Genese und Struktur des bürokratischen Felds, in: Ders., Praktische Vernunft. Zur Theorie des Handelns, Frankfurt / Main, S. 91-136.

Denninger, Tina / Silke van Dyk / Stephan Lessenich / Anna Richter (2012): Vom „,verdienten Ruhestand“ zum „Alterskraftunternehmer“? Bilder des Alter(n)s im gesellschaftlichen Wandel nach dem Systemumbruch, in: Heinrich Best / Everhard Holtmann (Hrsg.), Aufbruch der entsicherten Gesellschaft. Deutschland nach der Wiedervereinigung, Frankfurt / Main - New York / NY, S. 369-387.

Deutscher Bundestag (2006): Fünfter Bericht zur Lage der älteren Generation in der Bundesrepublik Deutschland und Stellungnahme der Bundesregierung, Drucksache 16 / 2190, Berlin.

Deutscher Bundestag (2010): Sechster Bericht zur Lage der älteren Generation in der Bundesrepublik Deutschland und Stellungnahme der Bundesregierung, Drucksache 17 / 3815, Berlin.

van Dyk, Silke / Stephan Lessenich (2009): „Junge Alte“: Vom Aufstieg und Wandel einer Sozialfigur, in: Dies. (Hrsg.), Die jungen Alten. Analysen einer neuen Sozialfigur, Frankfurt / Main - New York / NY, S. 11-48.

van Dyk, Silke / Stephan Lessenich / Tina Denninger / Anna Richter (2010): Die „Aufwertung“ des Alters. Eine gesellschaftliche Farce, in: Mittelweg 36 / 5, S. 15-33.

Esping-Andersen, Gøsta (1990): The Three Worlds of Welfare Capitalism, Cambridge.

Gilleard, Chris / Paul Higgs (2002): The third age: class, cohort or generation?, in: Ageing \& Society 22, S. 369-382.

Gilleard, Chris / Paul Higgs (2005): Contexts of Ageing. Class, Cohort and Community, Cambridge.

Göckenjan, Gerd (1993): Alter - Ruhestand - Generationsvertrag? Zum Altersdiskurs aus historischstruktureller Perspektive, in: Aus Politik und Zeitgeschichte B17, S. 3-10. 
Goodin, Robert / Julian LeGrand (1987): Not Only for the Poor. The Middle Classes and the Welfare State, London.

Graefe, Stefanie / Silke van Dyk (2012): Die Kategorie Geschlecht in der Konstruktion subjektiver Alterslosigkeit, in: Zeitschrift für Soziologie der Erziehung und Sozialisation 32, S. 154-171.

Graefe, Stefanie / Silke van Dyk / Stephan Lessenich (2011): Altsein ist später. Alter(n)snormen und Selbstkonzepte in der zweiten Lebenshälfte, in: Zeitschrift für Gerontologie und Geriatrie 44, S. 299-305.

Hockey, Jenny / Allison James (2003): Social Identities across the Life Course, Basingstoke - New York / NY.

Jones, Rebecca L. (2006): „Older people“ talking as if they are not older people: Positioning theory as an explanation, in: Journal of Aging Studies 20, S. 79-91.

Kaufman, Sharon R. (1986): The ageless self. Sources of meaning in later life, London.

Kohli, Martin (1985): Die Institutionalisierung des Lebenslaufs. Historische Befunde und theoretische Argumente, in: Kölner Zeitschrift für Soziologie und Sozialpsychologie 37, S. 1-29.

Kohli, Martin / Harald Künemund / Andreas Motel-Klingebiel / Marc Szydlik (2005): Soziale Ungleichheit, in: Martin Kohli / Harald Künemund (Hrsg.), Die zweite Lebenshälfte. Gesellschaftliche Lage und Partizipation im Spiegel des Alters-Survey, 2. erweiterte Auflage, Wiesbaden, S. 318-336.

Kolland, Franz (2010): Altersbilder und ihre normative Wirkung im Wandel der Erwerbsarbeit, in: Kai Brauer / Wolfgang Clemens (Hrsg.), Zu alt? „Ageism“ und Altersdiskriminierung auf Arbeitsmärkten, Wiesbaden, S. 61-80.

von Kondratowitz, Hans-Joachim (2009): Alter(n) in Ost und West: Der Wandel normativer Modellierungen des Alter(n)s in historisch vergleichender Perspektive, in: Silke van Dyk / Stephan Lessenich (Hrsg.), Die jungen Alten. Analysen einer neuen Sozialfigur, Frankfurt / Main - New York / NY, S. 256-278.

Kuchler, Barbara (2006): Bourdieu und Luhmann über den Wohlfahrtsstaat. Die Autonomie gesellschaftlicher Teilbereiche und die Asymmetrie der Gesellschaftstheorie, in: Zeitschrift für Soziologie 35, S. 5-23.

Lampert, Thomas / Anke-Christine Saß / Michael Häfelinger / Thomas Ziese (2005): Armut, soziale Ungleichheit und Gesundheit. Expertise des Robert Koch-Instituts zum 2. Armuts- und Reichtumsbericht der Bundesregierung, Berlin.

Lawler, Stephanie (2005): Disgusted subjects: the making of middle-class identities, in: The Sociological Review 53, S. 429-446.

Lessenich, Stephan (2008): Die Neuerfindung des Sozialen. Der Sozialstaat im flexiblen Kapitalismus, Bielefeld.

Lessenich, Stephan (2009a): Das Elend der Mittelschichten. Die „Mitte“ als Chiffre gesellschaftlicher Transformation, in: Widersprüche 111, S. 19-28.

Lessenich, Stephan (2009 b): Lohn und Leistung, Schuld und Verantwortung. Das Alter in der Aktivgesellschaft, in: Silke van Dyk / Stephan Lessenich (Hrsg.), Die jungen Alten. Analysen einer neuen Sozialfigur, Frankfurt / Main - New York / NY, S. 279-295.

Marshall, Thomas H. (1963 [1949]): Citizenship and Social Class, in: Ders., Sociology at the Crossroads and Other Essays, London, S. 67-127.

McMullin, Julie Ann / John Cairney (2004): Self-esteem and the intersection of age, class, and gender, in: Journal of Aging Studies 18, S. 75-90.

Münch, Richard (2009): Von der konsumtiven zur produktiven Sozialpolitik: Paradigmenwechsel der Sozialordnung?, in: Ders., Das Regime des liberalen Kapitalismus. Inklusion und Exklusion im neuen Wohlfahrtsstaat, Frankfurt / Main - New York / NY, S. 135-185.

Nikander, Pirjo (2009): Doing change and continuity: age identity and the micro-macro divide, in: Ageing \& Society 29, S. 863-881. 
Nolte, Paul (2004): „Fürsorgliche Vernachlässigung“? Umrisse einer neuen Politik der Unterschichten, in: Ders., Generation Reform. Jenseits der blockierten Republik, München, S. 57-73.

Pietilä, Ilkka / Hanna Ojala (2011): Acting age in the context of health: Middle-aged working class men talking about bodies and ageing, in: Journal of Aging Studies 25, S. 380-389.

Richter, Matthias / Klaus Hurrelmann (2007): Warum die gesellschaftlichen Verhältnisse krank machen, in: Aus Politik und Zeitgeschichte B42, S. 3-10.

Rieger, Elmar (2003): Bürger: Kulturelle Grundlagen des demokratischen Wohlfahrtsstaates, in: Stephan Lessenich (Hrsg.), Wohlfahrtsstaatliche Grundbegriffe. Historische und aktuelle Diskurse, Frankfurt / Main - New York / NY, S. 215-242.

Schommer, Martin (2008): Wohlfahrt im Wandel. Risiken, Verteilungskonflikte und sozialstaatliche Reformen in Deutschland und Großbritannien, Wiesbaden.

Siri, Jasmin (2010): Die Halbierung der Bürgerlichkeit. Zur Form „,neo-bürgerlicher“ Diskurse in der massenmedialen Öffentlichkeit, in: PROKLA 160, S. 325-340.

Skeggs, Beverly (2004): Class, Self, Culture, London - New York / NY.

de Swaan, Abram (1993): Der sorgende Staat. Wohlfahrt, Gesundheit und Bildung in Europa und den USA der Neuzeit, Frankfurt / Main - New York / NY.

Thompson, Edward P. (1980): Die ,moralische Ökonomie‘ der englischen Unterschichten im 18. Jahrhundert, in: Ders., Plebeische Kultur und moralische Ökonomie. Aufsätze zur englischen Sozialgeschichte des 18. und 19. Jahrhunderts, Frankfurt / Main, S. 67-130.

Vester, Michael (2005): Die selektive Bildungsexpansion. Die ständische Regulierung der Bildungschancen in Deutschland, in: Peter A. Berger / Heike Kahlert (Hrsg.), Institutionalisierte Ungleichheiten. Wie das Bildungswesen Chancen blockiert, Weinheim - München, S. 39-70.

Vester, Michael / Peter von Oertzen / Heiko Geiling / Thomas Hermann / Dagmar Müller (2001): Soziale Milieus im gesellschaftlichen Strukturwandel. Zwischen Integration und Ausgrenzung, 2. Auflage, Frankfurt / Main.

Vogel, Berthold (2009): Wohlstandskonflikte. Soziale Fragen, die aus der Mitte kommen, Hamburg.

Wacquant, Loïc J. D. (1991): Making Class: The Middle Class(es) in Social Theory and Social Structure, in: Scott G. McNall / Rhonda F. Levine / Rick Fantasia (Hrsg.), Bringing Class Back In. Contemporary and Historical Perspectives, Boulder / CO, S. 39-64.

Wahl, Anke (2003): Die Veränderung von Lebensstilen. Generationenfolge, Lebenslauf und sozialer Wandel, Frankfurt / Main - New York / NY.

Dr. Stefanie Graefe

Prof. Dr. Stephan Lessenich Friedrich-Schiller-Universität Jena Institut für Soziologie Carl-Zeiß-Straße 2 07743 Jena

stefanie.graefe@uni-jena.de stephan.lessenich@uni-jena.de 